\title{
Role of Transpedicular Percutaneous Vertebral Biopsy for Diagnosis of Pathology in Vertebral Compression Fractures
}

\author{
Saurabh Shrinivas Pagdal ${ }^{1}$, Sunil Nadkarni ${ }^{1}$, Sharad Moreshwar Hardikar ${ }^{2}$, Madan Sharad Hardikar ${ }^{1}$ \\ ${ }^{1}$ Department of Orthopedic Surgery, Hardikar Hospital, Pune, India \\ ${ }^{2}$ Head of Institutet, Hardikar Hospital, Pune, India
}

\section{Study Design: Retrospective observational study.}

Purpose: To identify the role of percutaneous vertebral biopsy in histopathological diagnosis of vertebral compression fractures and to identify the frequency of unexpected malignancy in vertebral compression fractures.

Overview of Literature: Vertebral compression fractures are common in the Indian population. Magnetic resonance imaging and nuclear imaging have some limitations in the diagnosis of definitive pathology of vertebral compression fractures. Therefore, histological confirmation is necessary for definitive diagnosis and to plan appropriate management for patient.

Methods: A retrospective observational study was conducted involving 84 patients who underwent percutaneous vertebral biopsy between 2010 and 2014. We performed C-arm guided percutaneous transpedicular core vertebral biopsy of vertebral compression fractures under combination of local anesthesia and intravenous conscious sedation.

Results: Sufficient biopsy material was obtained in 79 of the 84 cases. In the other five cases, biopsy material was not sufficient for reporting. Out of the 79 cases, osteoporotic pathology was detected in 69 patients, malignancy was detected in 8 patients and no pathology was found in 2 patients. Two patients with distant metastases to vertebra were identified. Primary spinal malignancy was detected in 6 patients (1 unsuspected plasmacytoma, 5 diagnosed malignancy preoperatively). So, the frequency of unsuspected malignancy of this study was 1.19\% (1/84). None of the patients had any complications.

Conclusions: $\mathrm{C}$-arm guided percutaneous transpedicular vertebral biopsy is useful in obtaining definitive histopathological diagnosis of vertebral compression fractures, especially in differentiating malignant and non-malignant vertebral compression fractures and helping plan appropriate management of patients. The rate of unexpected malignancy in vertebral compression fracture was 1.19\%.

Keywords: Needle biopsies; Compression fracture; Osteoporosis; Second malignancies

\section{Introduction}

Vertebral compression fractures (VCFs) are characterized by loss of vertebral height anteriorly, with no loss of posterior vertebral height [1]. Height reduction of the ver- tebral body should be $20 \%$ or at least $4 \mathrm{~mm}$ [2]. Most of the compression fractures are asymptomatic and go unnoticed. Compression fractures are noticed after severe back pain. Functional limitations, spinal instability or kyphotic deformity can be associated with compression fractures.

Received Jan 12, 2016; Revised Jan 30, 2016; Accepted Feb 21, 2016

Corresponding author: Saurabh Shrinivas Pagdal

Academic Department, Hardikar Hospital, University road, Shivajinagar, Pune, Maharashtra 411005, India

Tel: +91-99-7564-1564, Fax: +91-020-2553-5632, E-mail: saurabhpagdal19@gmail.com 
Vertebral compression fractures are common in Indian population, with $15 \%-20 \%$ of older urban adults aged over 50 years showing evidence of at least one vertebral fracture [3]. The prevalence of radiographic vertebral fractures in older adults in Delhi was recently reported to be $17.9 \%$ (18.8\% in males and $17.1 \%$ in females) indicating that vertebral fracture prevalence in India is similar to Western populations [4].

Diagnosis of VCFs is not difficult. The real problem is diagnosis of the definitive cause of VCF. Various causes of VCF include low bone density, osteomalacia, malignant lesions or infectious bone lesions [5]. Magnetic resonance imaging (MRI) is used to differentiate between malignant and benign VCFs [6]. But, there are some disadvantages of MRI in determining the cause of VCF. Overlap between the MRI characteristics of non-malignant and malignant VCFs makes preoperative diagnosis difficult [7]. Changes in observer performance also change the diagnosis [6]. Patients with known malignancy can also develop osteoporotic VCFs as both malignancy and osteoporosis can co-exist [8]. Therefore, histological confirmation is necessary for definitive diagnosis and to plan appropriate management for patient.

We performed C-arm guided percutaneous transpedicular core vertebral biopsy of vertebral compression fractures. The objectives of this study are to identify the role of percutaneous vertebral biopsy in definitive histopathological diagnosis of vertebral compression fractures and its effect on future treatment plan, and to identify the frequency of unexpected malignancy in vertebral compression fractures.

\section{Materials and Methods}

\section{Data collection}

This is an institution based retrospective observational study involving 84 patients who underwent percutaneous vertebral biopsy. The study was conducted between 2010 and 2014

\section{Inclusion and exclusion criteria}

The inclusion criterion was patients with vertebral compression fracture. Exclusion criteria were known bleeding disorder, pregnancy and intracranial space occupying lesion. Results of data analysis are presented as numbers and percentages.

\section{Surgical techniques}

Each patient was placed prone on regular table. Intravenous conscious sedation was given. Level of biopsy was marked using a metallic object under the C-arm guidance. One $\mathrm{cm}$ wheal was raised at the skin entry site using a 25 -gauge needle and a local anesthetic agent (2\% lignocaine). The local anesthetic was administered into the deeper soft tissues and periosteum of vertebra under image guidance of C-Arm. A scalpel blade was used to make a dermatology incision at the skin entry site. A Jamshidi needle was then advanced up to the medial pedicular line seen in the anteroposterior view of the lumbar spine. For entry into the vertebral body through the pedicle, images were taken in the lateral view. When the needle tip was in a satisfactory position, the needle moved slightly in situ so that the bone sample could be collected easily. Core biopsy samples were sent for histopathological examination in a sterile container. Sometimes osseous blood was collected in a syringe after applying negative pressure. It was considered to be a biopsy specimen and was submitted for pathological analysis, since it is possible to diagnose malignancy from such tissue.

\section{Results}

There were 84 patients in the study conducted from 2010 to 2014. All the patients were included as per the inclusion and exclusion criteria. All biopsies were performed under combination of local anesthesia and intravenous sedation. All biopsies were performed with the patient in the prone position, using a Jamshidi needle. Fifty of the 84 patients (59.2\%) were females and 34 (40.48\%) were males. The mean age was 66.3 years. Out the 84 patients, biopsy samples were taken from the thoracic region in 28 (33.33\%) patients, from the lumbar region in 37 (44.05\%) patients and from both lumbar and thoracic regions in $19(22.62 \%)$ patients. In 48 patients (57.14\%) biopsy samples were taken from only one vertebra and in 36 patients $(42.86 \%)$ biopsy samples were taken from more than one vertebra. Sufficient biopsy material was obtained in 79 cases. In the other five cases, biopsy material was not sufficient for reporting. Of the 79 cases, osteoporotic pathology was detected in 69 patients, malignancy was detected in 8 patients and no pathology was found in 2 
patients. Two patients with distant metastases to vertebra were identified. Primary spinal malignancy was detected in 6 patients ( 1 unsuspected plasmacytoma, 5 diagnosed malignancy preoperatively) (Fig. 1). The frequency of unsuspected malignancies was $1.19 \%(1 / 84)$. None of the patients had any complications. Concerning primary spinal malignancy, out of 6 patients, 5 were diagnosed with multiple myeloma. One case was diagnosed with plasmacytoma, which was unsuspected. Concerning metastatic VCFs, the metastases were pathological in 2 cases. Primary malignancies were adenocarcinoma of lung and adenocarcinoma of prostate.

\section{Discussion}

There are various modalities for diagnosis of the cause of VCFs. MRI can differentiate between malignant and benign VCFs [6]. Malignant VCFs are suspected when the findings present on imaging include convex contour of the posterior vertebral border, epidural masses and infiltration of the vertebral posterior elements [5,9]. Posterior bone fragment retropulsion with normal bone marrow and accumulation of fluid within vertebral bodies are seen in osteoporotic VCFs [5,10]. Overlapping between the MRI imaging characteristics of non-malignant and malignant VCFs makes preoperative diagnosis difficult [7]. Changes in observer performance also change the diagnosis while assessing the MRI [6].

Bone scintigraphy is used for screening for skeletal tu- mors. The tracer accumulates in the reactive bone which is formed in response to the lesion. False-negative results are common in multiple myeloma, leukemia and anaplastic carcinomas. Because tracer accumulation may occur at any skeletal site with an elevated rate of bone turnover, radionuclide uptake may be nonspecific and may accompany trauma and infection [11]. Also, nuclear imaging studies are costly. Thus, bone scan cannot be used in every VCF patient.

For planning future treatment plan, knowledge of the pathology of VCFs is essential. Vertebral biopsy is important in identifying unsuspected pathology. There are various primary tumors of the vertebral body that occur in middle aged to senile aged patients. Primary benign tumor includes hemangioma; primary malignant tumors include chondrosarcoma, malignant fibrous histiocytoma, multiple myeloma, plasmacytoma and chordoma [12]. A 2013 study evaluated 450 patients and identified 5 (1.11\%) unsuspected malignancies including 3 patients with spinal metastases and 2 patients with multiple myeloma [5]. In the study performed by Zhang et al. [13], the rate of unsuspected malignancy was $0.4 \%(2 / 546)$ including one patient with metastatic carcinoma and one patient with multiple myeloma. Another study [14] evaluated 103 patients and reported a $2.9 \%(3 / 103)$ rate of unsuspected malignancy. In another study [15], the rate of unsuspected malignancy was $3.8 \%$ (3/78). In our study, the rate of unsuspected malignancy was $1.19 \%$ (1/84) including one patient with plasmacytoma. Diagnosis of plasmacytoma

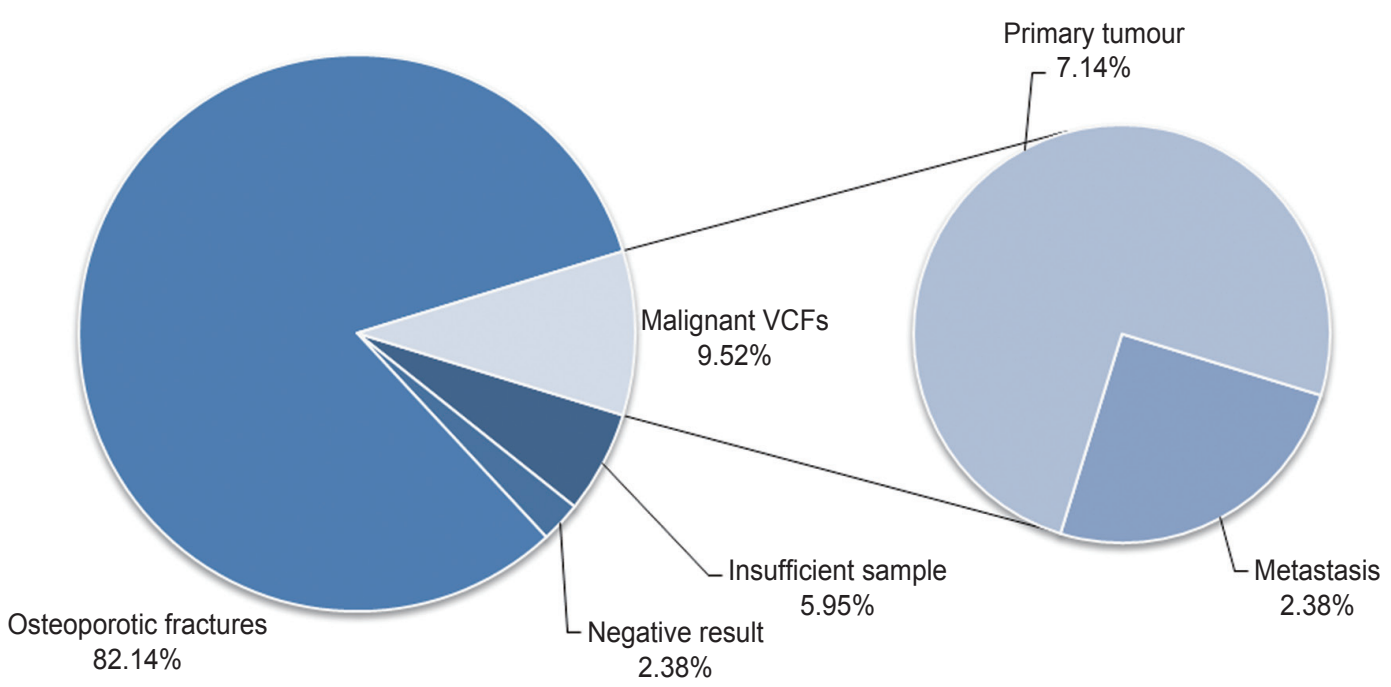

Fig. 1. Pie chart of biopsy results of vertebral compression fractures (VCFs). 
was further confirmed by immunohistochemistry in that patient.

Diagnosis of malignant VCF is confirmed by histopathological examination of biopsy sample. Vertebral biopsy detects type and grade of malignancy. This facilitates proper management of the pathology. In one study, malignant VCFs were detected in 61 patients out of 450 patients [5]. In our study, malignancy was detected in 8 patients out of 84 patients.

There were several limitations in this study. It was a retrospective observational study. The number of cases $(n=84)$ is less compared to other studies $[5,13]$. The relationship between osteoporosis and VCFs was not analyzed. The diameter and type of biopsy needle was not investigated.

In our study, biopsy material was not sufficient for reporting in 5 of 84 cases. In a prior study [15], 7 of 78 biopsies could not be interpreted as a result of suboptimal quality biopsy material. Mukherjee et al. [2] performed 184 biopsies in 135 patients. Of these biopsies, $78 \%$ were adequate diagnostic specimens and 41 (22\%) were inadequate and therefore inconclusive. In patients with insufficient biopsy sample, the samples contained scant bone tissue with contamination of disc materials in 3 cases, likely because of incorrect routing of the biopsy needle. All 5 cases had single level VCF with significant decrease in vertebral body height. In multiple level VCFs cases, we tried to get biopsy sample from more than 1 level. In tumors, the necrotic or cystic areas should be avoided and the radiologist should try to identify these areas on computed tomography or MR images prior to biopsy [16]. The authors also also suggested that a pathologist should be present during the procedure to determine the adequacy of the tissue specimen. But, it is not always possible for a pathologist to present at every procedure, especially in smaller centers.

\section{Conclusions}

C-arm guided percutaneous transpedicular vertebral biopsy is useful in obtaining definitive histopathological diagnosis of vertebral compression fractures, and especially in differentiating malignant and non-malignant VCFs. This helps in planning appropriate management for patients. The rate of unexpected malignancy in vertebral compression fracture was $1.19 \%$.

\section{Conflict of Interest}

No potential conflict of interest relevant to this article was reported.

\section{References}

1. Williams KD. Fractures, dislocations, and fractures: dislocation of the spine. In: Canale ST, Beaty JH, editors. Campbell's operative orthopedics. 12th ed. Vol.2. Canada: Elsevier Mosby; 2013. p.1608-9.

2. Mukherjee S, Thakur B, Bhagawati D, et al. Utility of routine biopsy at vertebroplasty in the management of vertebral compression fractures: a tertiary center experience. J Neurosurg Spine 2014;21:687-97.

3. Mithal A, Bansal B, Kyer CS, Ebeling P. The Asia-Pacific Regional Audit: epidemiology, costs, and burden of osteoporosis in India 2013: a report of International Osteoporosis Foundation. Indian J Endocrinol Metab 2014;18:449-54.

4. Marwaha RK, Tandon N, Gupta Y, et al. The prevalence of and risk factors for radiographic vertebral fractures in older Indian women and men: Delhi Vertebral Osteoporosis Study (DeVOS). Arch Osteoporos 2012;7:201-7.

5. Chou KN, Lin BJ, Chien LY, Tsai WC, Ma HI, Hueng DY. Simple transpedicular vertebral biopsy for diagnosis of malignancy in vertebral compression fracture. Neurol India 2013;61:587-92.

6. Thawait SK, Marcus MA, Morrison WB, Klufas RA, Eng J, Carrino JA. Research synthesis: what is the diagnostic performance of magnetic resonance imaging to discriminate benign from malignant vertebral compression fractures? Systematic review and metaanalysis. Spine (Phila Pa 1976) 2012;37:E736-44.

7. An HS, Andreshak TG, Nguyen C, Williams A, Daniels D. Can we distinguish between benign versus malignant compression fractures of the spine by magnetic resonance imaging? Spine (Phila Pa 1976) 1995;20:1776-82.

8. Muthukumar N. Biopsy of osteoporotic vertebral compression fractures. Neurol India 2013;61:569-71.

9. Jung HS, Jee WH, McCauley TR, Ha KY, Choi KH. Discrimination of metastatic from acute osteoporotic compression spinal fractures with MR imaging. Radiographics 2003;23:179-87.

10. Baur A, Stabler A, Arbogast S, Duerr HR, Bartl R, 
Reiser M. Acute osteoporotic and neoplastic vertebral compression fractures: fluid sign at MR imaging. Radiology 2002;225:730-5.

11. Shah LM, Salzman KL. Imaging of spinal metastatic disease. Int J Surg Oncol 2011;2011:769753.

12. Fuchs B, Boos N. Primary tumors of the spine. In: Fuchs B, Boos N, editors. Spinal disorders: fundamentals of diagnosis and treatment. Berlin: Springer; 2008. p.951-76.

13. Zhang L, Li J, Yang H, Luo Z, Zou J. Histological evaluation of bone biopsy results during PVP or PKP of vertebral compression fractures. Oncol Lett 2013;
5:135-8.

14. Li Q, Hua S, Wang C, Cai S, Zhang J. The value of routine biopsy during percutaneous kyphoplasty for vertebral compression fractures. PLoS One 2014;9: e115417.

15. Muijs SP, Akkermans PA, van Erkel AR, Dijkstra $\mathrm{SD}$. The value of routinely performing a bone biopsy during percutaneous vertebroplasty in treatment of osteoporotic vertebral compression fractures. Spine (Phila Pa 1976) 2009;34:2395-9.

16. Peh W. CT-guided percutaneous biopsy of spinal lesions. Biomed Imaging Interv J 2006;2:e25. 